\title{
Optimierung und Klinische Entscheidungsunterstützung
}

\author{
Sebastian Sager
}

\begin{abstract}
Mediziner müssen täglich viele wichtige Entscheidungen treffen. Ein klinisches Beispiel ist die Planung und Dosierung einer Chemotherapie. Ein zweites Beispiel ist die Unterscheidung zwischen verschiedenen Herzrhythmusstörungen basierend auf EKG-Daten. Das notwendige (oftmals unbewusste) Expertenwissen ist nicht leicht transferierbar und beispielsweise im ländlichen Raum nicht immer verfügbar. Wir erläutern, wie durch eine Kombination von Mathematik, modelliertem Expertenwissen und klinischen Daten neuartige automatisierte Systeme zur Entscheidungsunterstützung entstehen.
\end{abstract}

\section{Einleitung}

Die mathematische Optimierung, flankiert durch Modellierung und Simulation, ist eine Schlüsseltechnologie unserer modernen Gesellschaft. Es gibt eine immense Vielfalt von Anwendungsgebieten, die durch Optimierung profitiert haben. Sie wurde für Design, Layout, Betrieb, Kalibrierung und Analysen von Produkten, Netzwerken, Prozessen und Systemen eingesetzt und ist der Kern moderner Methoden der Steuerung und des Maschinellen Lernens. Auch in der Medizin haben mathematische Ansätze zu großen Fortschritten geführt. So wurden beispielsweise durch systembiologische Ansätze zelluläre Pfadwege besser verstanden. Moderne Algorithmen werden auch auf einer höheren Ebene eingesetzt, so beispielsweise in der Krankenhauslogistik, bei der Magnetresonanztomographie, der Dosierung von Insulin, oder der Kalibrierung von Bestrahlungen. Der Einfluss der Angewandten Mathematik auf unser modernes Leben ist gigantisch: in den Büchern $[4,10,14]$ sind einige Erfolgsgeschichten nachzulesen.

Interessanterweise haben fast alle Anwendungsgebiete ihrerseits mathematische Forschung stimuliert. Das Ziel dieses Artikels ist es, aufzuzeigen inwieweit auch die Entscheidungsunterstützung in der klinischen Praxis von Optimierung profitieren kann und ihrerseits neue Herausforderungen an die Angewandte Mathematik liefert. Dieses wird beispielhaft an aktuellen Forschungsfragen des Projektes „Mathematical Optimization for clinical DEcision Support and Training" (MODEST) geschehen, das vom European Research Council (ERC) als Consolidator Grant von 2015-2020 gefördert wird. Im ERC Grant MODEST werden Krankheitsdynamiken modelliert und untersucht, inwieweit Optimierung dieser mathematischen Modelle zur Entscheidungsunterstützung bei Diagnose und Therapieplanung, aber auch analog zu Flugsimulatoren für die Ausbildung von Medizinern genutzt werden kann.

Es gibt zwei Kernbehauptungen und take-away Botschaften für die Leser/innen. Erstens geben, trotz der offensichtlichen Komplexität, Diversität und Unsicherheit menschlicher Körper und dessen was in ihnen vorgeht, überraschend einfache mathematische Modelle in Kombination mit Optimierung sehr hilfreiche Einblicke. Diese Modelle kapseln die wesentlichen dynamischen Charakteristiken und basieren auf medizinischem Expertenwissen, sind aber gleichzeitig so einfach gehalten, dass sie sich für Personalisierungen und einen Einsatz in Echtzeit eignen. Ich glaube, dass diese Ansätze für bestimmte Fragestellungen besser sind als das, was man praktisch mit reinen datengetriebenen Ansätzen des Maschinellen Lernens erreichen könnte.

Zweitens motivieren klinische Entscheidungen neue und interessante Klassen von Optimierungsproblemen. Eine besondere Herausforderung ist, dass die Bestimmung (oder Personalisierung) des mathematischen Modelles und seine Optimierung miteinander interagieren und zur gleichen Zeit erfolgen müssen. Überraschende Strukturen können sich ergeben, zum Beispiel wenn die Dimension der Optimierungsvariablen von der optimalen Lösung abhängt.

Wir betrachten nach kurzen allgemeinen Vorbemerkungen drei Fallstudien aus dem laufenden ERC Consolidator Grant MODEST, die alle auf engen Kooperationen mit medizinischen Partnern basieren. Natürlich ist dies nur eine sehr subjektive Auswahl. Es gibt viele weitere Erfolgsgeschichten in der Schnittmenge aus Optimierung und klinischer Entscheidungsunterstützung, die von anderen geschrieben wurden. Alle Beispiele werden einheitlich dargestellt mit klinischem Hintergrund, dem korrespondierenden Optimierungsproblem, Modellierungsaspekten, Herausforderungen und Lösungsansätzen sowie Ergebnissen. Zum Abschluss geben wir einen spekulativen Ausblick in die Richtung eines Modell-getriebenen Trainings von klinischer Entscheidungsfindung.

Vorbemerkungen zum wissenschaftlichen Rechnen

Zentrale Aspekte der Angewandten Mathematik sind die Modellierung, die Simulation und die Optimierung. Bei der Modellierung geht es darum, mathematische Ausdrücke für Fragestellungen aus der „echten Welt“ zu finden. Häufig sind dies Variablen bzw. Funktionen von Ort und Zeit, 
die beispielsweise Mengen, Positionen, Geschwindigkeiten, Konzentrationen, Lagerbestände, oder Temperaturen beschreiben. Oftmals unterscheidet man hier noch weiter zwischen Zuständen (abhängigen Variablen) und Steuerungen (unabhängigen Variablen). Die unabhängigen Variablen können von außen (also vom Menschen, oder von einem Algorithmus) beeinflußt und eingestellt werden, die abhängigen dagegen resultieren dann durch die Umsetzung in der realen oder der simulierten Welt: wenn Gaspedal und Bremse auf eine bestimmte Art und Weise bedient werden, dann folgen daraus die Geschwindigkeit und Position des Fahrzeugs. Zur Modellierung gehört aber auch die Formulierung von Gleichungen, die den Zusammenhang zwischen den verschiedenen Variablen und Funktionen beschreiben. Einige sind sehr gut verstanden und liefern sehr realistische mathematische Modelle ab - hierzu gehören beispielsweise das Newtonsche Kraftgesetz „Kraft ist Masse mal Beschleunigung“, Material- oder Impulserhaltungssätze oder Reaktionskinetiken. Andere haben einen eher spekulativen Charakter und es ist nicht immer klar, ob die Realität wirklich exakt wiedergegeben wird - hierzu gehören beispielsweise Zusammenhänge aus den Wirtschafts- und Finanzwissenschaften oder wie in unserem Kontext den Lebenswissenschaften. Auch wenn hier keine sogenannten first principles bekannt (oder noch umstritten) sind, so sind durch iterative Vergleiche mit Messdaten aus der echten Welt erhebliche Fortschritte erzielt worden. Und - wie wir weiter unten sehen werden - oftmals ist es für praktische Zwecke vollkommen ausreichend, phänomenologische Modelle zu verwenden, solange diese nur die wesentlichen Charakteristika hinreichend genau wiedergeben.

Bei der Simulation geht es darum, die formulierten Gleichungssysteme zu lösen. Dieses ist oftmals nicht analytisch möglich, weswegen man die Lösungen durch numerische Rechnungen auf dem Rechner mit möglichst genauen Fehlerschranken annähert. Man bekommt als Lösung also keinen geschlossenen mathematischen Ausdruck (z. B. eine Funktion der Zeit), sondern Zahlenwerte auf einem Gitter. Die Kombination aus Modellierung und Simulation erlaubt nun schon Vorhersagen für die Zukunft zu machen: was wäre wenn? Die vielleicht prominenteste Anwendung sind die sogenannten Crashtests, bei denen bis in die 199oer Jahre hinein extrem teure Auto-Prototypen vor Wände gefahren wurden, um die Auswirkungen von Designentscheidungen am kaputten Auto zu untersuchen. Heutzutage werden allenfalls noch fertig entwickelte Prototypen, die schon tausende von virtuellen Crashtests überstanden und Millionen von virtuellen Kilometern gefahren sind, zur Bestätigung der schon bekannten Ergebnisse in der Realität gecrasht.

Die Optimierung setzt nun dort an, wo das reine Versuch-und-Irrtum Konzept an seine Grenzen stößt - weil es (selbst für das rasend schnelle Ausprobieren einzelner Möglichkeiten auf dem Computer) zu viele Möglichkeiten gibt und diese auf sehr nicht-intuitive und komplexe Art und Weise miteinander verknüpft sein können. Zuerst müssen eine Zielfunktion (was soll minimiert oder maximiert werden?) und Nebenbedingungen (Auto soll auf der StraBe bleiben, das Immunsystem intakt bleiben, die Temperatur einen kritischen Wert nicht überschreiten) formuliert werden. Für die entstehenden mathematischen Optimierungsprobleme werden seit den 1940er Jahren intelligente Algorithmen entwickelt und auf Computern eingesetzt, um beweisbar optimale Lösungsstrategien $\mathrm{zu}$ finden. In den folgenden Abschnitten werden optimale Lösungen eingesetzt um inverse Simulationen (was sind die Ursachen von beobachtetem Verhalten?) durchzuführen, Verbesserungspotentiale von Therapien zu analysieren und quantifizieren, konkrete Therapiepläne zu berechnen, oder um die Ausbildung im Bereich Komplexes Problemlösen zu verbessern.

\section{Inverse Simulation von Herzrhythmusstörungen}

\section{Klinischer Hintergrund und Optimierungsfragen}

Es gibt mehrere Dutzend unterschiedliche Ursachen von Herzrhythmusstörungen. Während die meisten von ihnen problemlos von Experten unterschieden werden können, stellt die Unterscheidung zwischen Vorhofflimmern (AFib) und regulären Vorhofarrhytmien inklusive Vorhofflattern (AFlu) und fokalen Vorhoftachykardien eine diagnostische Herausforderung dar. Die normalerweise verfügbare Datenbasis, ein Oberflächen-Elektrokardiogramm (EKG), sieht für beide Fälle sehr ähnlich aus und stellt Laien, Mediziner und Computeralgorithmen gleichermaßen vor Probleme. Fehlinterpretationsraten von bis $\mathrm{zu} 80 \%$ werden in der Literatur berichtet [22], was angesichts der unterschiedlichen durch die Diagnose indizierten Behandlungen (häufig Antiarrhytmika bei AFib und Ablation bei AFlu) unerwünschte Folgen hat. Zudem nimmt die Häufigkeit von atypischen Formen von AFlu durch Komplikationen von Ablationsprozeduren im linken Vorhof zu.

Das elektrische Schrittmachersignal aus dem Sinusknoten ist ursächlich für das spätere mechanische Pumpen des Herzens. Es pflanzt sich durch die Vorhöfe und den Atrioventrikulärknoten (AV Knoten) bis zu den ventrikulären Kammern fort. Bei AFib ist schon die elektrische Aktivierung der Vorhöfe chaotisch und intrinsisch irregulär. Bei AFlu dagegen ist die Aktivierung der Vorhöfe regulär, d. h., sie erfolgt in konstanten Abständen. Dieses reguläre Signal führt aufgrund eines komplexen Blockierungsmechanismus im AV Knoten ebenfalls zu einer irregulären Aktivierung der Ventrikel. Während es theoretisch ein leichtes sein sollte, die unterschiedlichen Aktivierungen der Vorhöfe zu unterscheiden, sind diese aufgrund der niedrigen Voltzahlen in der Praxis kaum vom Grundrauschen des Signals zu unterscheiden. Zusammengefasst stehen wir also vor einer Situation, in der nur die Zeitpunkte der deutlich erkennbaren $\mathrm{R}$ Wellen (den Pieptönen eines Herzratenmonitors) in denen die Ventrikel aktiviert werden, als input für die Diagnose zur Verfügung stehen. Wir setzen weiterhin voraus, dass eine vorgeschaltete Diagnose schon sichergestellt hat, dass entweder AFib oder AFlu für die beobachtete Arrhytmie verantwortlich sind.

Wir benutzen ein mathematisches Modell für das komplexe, aber deterministische Blockierungsverhalten im AV Knoten und Optimierung, um dieses Modell basierend auf Input Daten zu personalisieren. Einen kleinen Zielfunktionswert, d. h. eine gute Übereinstimmung zwischen der Si- 
mulation und den gemessenen Zeitpunkten, interpretieren wir als eine hohe Wahrscheinlichkeit für reguläres Verhalten (AFlu), während wir einen großen Zielfunktionswert als eine hohe Wahrscheinlichkeit für chaotisches Verhalten interpretieren, das nicht durch das Modell erklärt werden kann (AFib).

Mathematische Modellierung der Dynamik und des Optimierungsproblems

Viele unterschiedliche Algorithmen zur Analyse von Herzrhythmusstörungen wurden vorgeschlagen, wie Fouriertransformationen, Wavelets, Clustern von RR Zeiten, Maschinelles Lernen, Zelluläre Automaten, oder Nichtlineare Zeitreihenanalyse. Keiner dieser Ansätze konnte jedoch zufriedenstellende Ergebnisse für unser Problem und kurze EKGs liefern [15]. Auch eine Vielzahl mathematischer Modelle für das elektrische Reizleitungssystem des Herzens wurden vorgeschlagen. Das prominenteste von ihnen ist die Anpassung von Differentialgleichungen durch Noble [16], für die Hodgkin und Huxley 1963 den Medizin-Nobelpreis erhielten. In diesem Modell verändert sich das elektrische Potential durch die Zellmembran aufgrund von Ionenströmen, bei denen Sodium, Calcium und Potassium eine wichtige Rolle spielen. Ein Differentialgleichungssystem mit vier differentiellen Zuständen und mehreren geschätzten Modellparametern war in der Lage, bis dato unbekannte Phänomene vorherzusagen und führte zu vielen Erweiterungen, wie im Übersichtsartikel [17] beschrieben wird.

Eine Analyse des first-principle Modelles für das Verhalten auf zellulärer Ebene zeigt periodische, stabile Lösungen. Änderungen in Modellparametern, wie man sie von einem Patienten zum nächsten vermuten würde, ändern nicht das qualitative Verhalten der simulierten Ionenflüsse. Diese Flüsse kann man in Verbindung setzen zu sogenannten Refraktärzeiten, in denen die Zelle noch nicht wieder in der Lage ist ein weiteres elektrisches Signal weiterzugeben. Änderungen in Modellparametern führen allerdings zu modifizierten Zeitfenstern. Daher haben wir uns dafür entschieden, uns auf einfachere mathematische Modelle zu konzentrieren, in denen die Länge der Refraktärzeiten von größeren Zellverbünden im AV Knoten direkt modelliert wird, und Details über die unterliegende Zelldynamik vernachlässigt werden.

Wir entwickelten ein Modell mit mehreren Ebenen, das auf phänomenologischen Beobachtungen basiert. Es nutzt Filter vom Typ $n+1: n$, d. h. von $n+1$ ankommenden Signalen werden genau $n$ durch eine Ebene des AV Knotens weitergeleitet. Die Reisezeit ist dabei variabel und kann linear von Signal zu Signal ansteigen, bevor sie nach dem $n+1$.ten Signal zurück gesetzt wird. Aus der Sicht der unterliegenden zellulären Prozesse entspricht dies Signalen, die die Zelle zu unterschiedlichen Zeitpunkten der stabilen Oszillationen erreichen. Die Zeiten werden unter der vereinfachenden Annahme einer linearen Modifikation modelliert als

$$
x_{i}^{l}=x_{j}^{l-1}+\tau_{\text {const }}^{l}+k_{i, j}^{l} \tau_{\text {lin }}^{l}
$$

wobei $l$ der Blockierungslevel, $i$ die Nummer des ausgehenden Signals, und $j$ die Nummer des weitergeleiteten Signals ist. Die Zeiten $\tau_{\text {const }}^{l}$ und $\tau_{\text {lin }}^{l}$ sind Modellparameter und damit Freiheitsgrade der inversen Simulation. Der Zähler $k_{i, j}^{l}$ erhöht sich $n$ mal, bevor er auf 0 zurück gesetzt wird.

Unter der Annahme eines regulären (AFlu) Verhaltens gibt der Zielfunktionswert die Abweichung des simulierten Signales von den echten Messungen (R Zeitpunkte) an. In einer geeigneten Norm minimiert man $\left\|x^{n_{l}}-\bar{x}\right\|$, wobei $\bar{x}$ die gemessenen Zeitpunkte der R Wellen sind. Der Vektor $x^{n_{l}}$ von Zeitpunkten auf der untersten Blockierungsebene $n_{l}$ ist das Ergebnis einer Vorwärtssimulation eines regulären, aber a priori unbekannten Signalvektors $x^{0}$ in den Vorhöfen. Das Optimierungsproblem kann wie folgt zusammen gefasst werden.

$$
\begin{aligned}
\min \left\|x^{n_{l}}-\bar{x}\right\| & \text { unter den Nebenbedingungen } \\
& - \text { Schranken und Ganzzahligkeit, } \\
& - \text { Signal } x^{n_{l}} \text { ist das Ergebnis einer } \\
& \text { Vorwärtssimulation nach (1), } \\
& - \text { das Eingangs-Signal } x^{0} \text { (Aktivie- } \\
& \text { rung der Vorhöfe) ist regelmäßig. }
\end{aligned}
$$

Die unabhängigen diskreten Variablen sind die Anzahl der Blockierungsebenen $n_{l}$ und die jeweiligen Werte $n$ die die $n+1: n$ Blockierungen auf der Ebene $l$ charakterisieren. Die unbhängigen kontinuierlichen Variablen sind die Transitionszeiten $\tau_{\text {const }}^{l}, \tau_{\text {lin }}^{l}$ und der Abstand zwischen zwei Aktivierungen im Vorhof, gegeben durch $\Delta x^{0}:=x_{i}^{0}-x_{i-1}^{0}$. Abhängige Variablen sind die Signalzeiten $x_{i}^{l}$ und die Zähler $k_{i, j}^{l}$.

In einer etwas komplizierteren Formulierung kann noch berücksichtigt werden, dass das Blockierungsverhalten in der Praxis zwischen $n+1: n$ und $n+2: n+1$ wechseln kann. Dies liegt daran, dass das Verhalten von der Frequenz der eingehenden Signale abhängig ist, und die Aktivierung der Vorhöfe auch bei kurzen EKGs häufig im Bereich einiger Millisekunden variiert.

\section{Herausforderungen an die Forschung im Bereich der Optimierung}

Für fixierte unabhängige Optimierungsvariablen (beispielsweise aus einer äußeren Optimierungsschleife) können die abhängigen Variablen und damit alle Beschränkungen und die Zielfunktion ausgewertet werden. Es ist jedoch keineswegs trivial, (2) in geschlossener, kompakter Form zu schreiben und mit einer Standard Mixed-integer nonlinear programming (MINLP) Software zu lösen. Es gibt mehrere Schwierigkeiten.

- Die Dimensionen der Vektoren $x^{l}$ hängen vom Wert der Optimierungsvariablen $\Delta x^{0}$ ab. Je kleiner $\Delta x^{0}$ ist, umso mehr Signale erreichen die nächste Ebene.

- In der Zielfunktion muss die $j$.te gemessene R Welle mit dem $j$.ten Signal verglichen werden, dass die Ebene $n_{l}$ erreicht. Ob einem Signal $x_{i}^{n_{l}}$ tatsächlich $i-j+1$ geblockte Signale vorausgehen, hängt allerdings von den Optimierungsvariablen ab. Eine Möglichkeit der Modellierung besteht in der Einführung einer Matrix $\Phi \in\{0,1\}^{m_{1} \times m_{2}}$ mit Einträgen $\Phi_{i, j}$ die genau dann 1 sind, wenn dies der Fall ist. Dann kann man die Zielfunktion schreiben als $\min \left\|\Phi x^{n_{l}}-\bar{x}\right\|$. Wie man die logischen Bedingungen effi- 
zient formuliert um $\Phi$ derart zu bestimmen, ist jedoch unklar, insbesondere weil $m_{1}$ von $\Delta x^{0}$ abhängig ist.

- Die Werte $x_{i}^{l}$ hängen von den diskreten Zählern $k_{i, j}^{l} \in$ $\{0, \ldots, n-1\}$ ab, wie man in (1) sieht. Diese Zähler werden durch das $n+1: n$ Blockierungsverhalten der Ebene $l$ bestimmt. Das allererste Signal einer Ebene hat einen unbekannten Zählerwert, während alle folgenden durch Augmentieren und Resetting bestimmt sind. Das Einführen von $k_{i, j}^{l}$ als Optimierungsvariablen führt zu einer nichtlinearen rechten Seite in (1).

- Die Zielfunktion ist keineswegs eindeutig. Wie viele Signale sollten verglichen werden (zu wenige können fast immer erklärt werden, bei zu vielen gibt es häufig Variationen in $\Delta x^{0}$ ), welche Norm sollte verwendet werden, wie sollen Unterschiede zwischen der Anzahl simulierter und gemessener R Wellen penalisiert werden?

Eine gute geschlossene Formulierung zu finden ist ein offenes Problem.

\section{Lösungsansatz}

Wir haben einen umfassenden Ansatz für klinische Entscheidungsunterstützung implementiert. Er beginnt mit automatischer Datengenerierung mit einem Smartphone. Unsere mobile App HEAT kann R Wellen Zeitpunkte aus EKG-Fotos oder den Pieptönen eines Herzmonitors extrahieren. Die Daten werden über eine sichere Verbindung auf unseren Rechenserver geschickt, wo ein Optimierungsproblem gelöst wird. Ergebnisse werden als PDF Dateien exportiert und zurück an die App geschickt.
Um das Optimierungsproblem effizient lösen zu können, haben wir einen maßgeschneiderten Branch and Bound Algorithmus implementiert. Der Einfluss des aktuellen Mehrebenen Blocks wird durch eine Vorwärtssimulation ausgewertet. Wir enumerieren die diskreten Variablen und die kontinuierlichen Variablen wie $\Delta x^{0}$ auf einem Millisekunden Gitter. Das erlaubt es, in einer gewissen Reihenfolge durch die Vorwärtssimulationen zu gehen und bestimmte Teilbäume zu verwerfen, wenn klinisch motivierte Beschränkungen oder logische Implikationen verletzt sind. Die Rechenzeiten für drei Blockierungsebenen und 22 R Wellen liegen bei ungefähr 1 bis 3 Sekunden auf einem Standardnotebook.

\section{(Vorläufige) Ergebnisse und Ausblick}

Abbildung 1 zeigt ein exemplarisches Ergebnis für AFlu. Es ist eines von 380 EKGs aus einer Benchmarkdatenbank, die wir gemeinsam mit unseren klinischen Partnern in Heidelberg erstellt haben. Alle davon haben den höchstmöglichen Goldstandard, Experten-interpretierte intrakardiale Messungen. Solche Messungen sind nur bei Operationen am offenen Herzen, und nicht unter normalen klinischen Bedingungen verfügbar. Unser optimierungsgetriebener Ansatz resultiert in einer Sensitivität (Prozentzahl der AFlu Fälle die als solche erkannt werden) von 0.81 und einer Spezifität (Prozentzahl der AFib Fälle die als solche erkannt werden) von 0.87 . Wenn man den Hauptindikator für diagnostische Tests, den Area Under the Curve-Wert der Receiver Operating

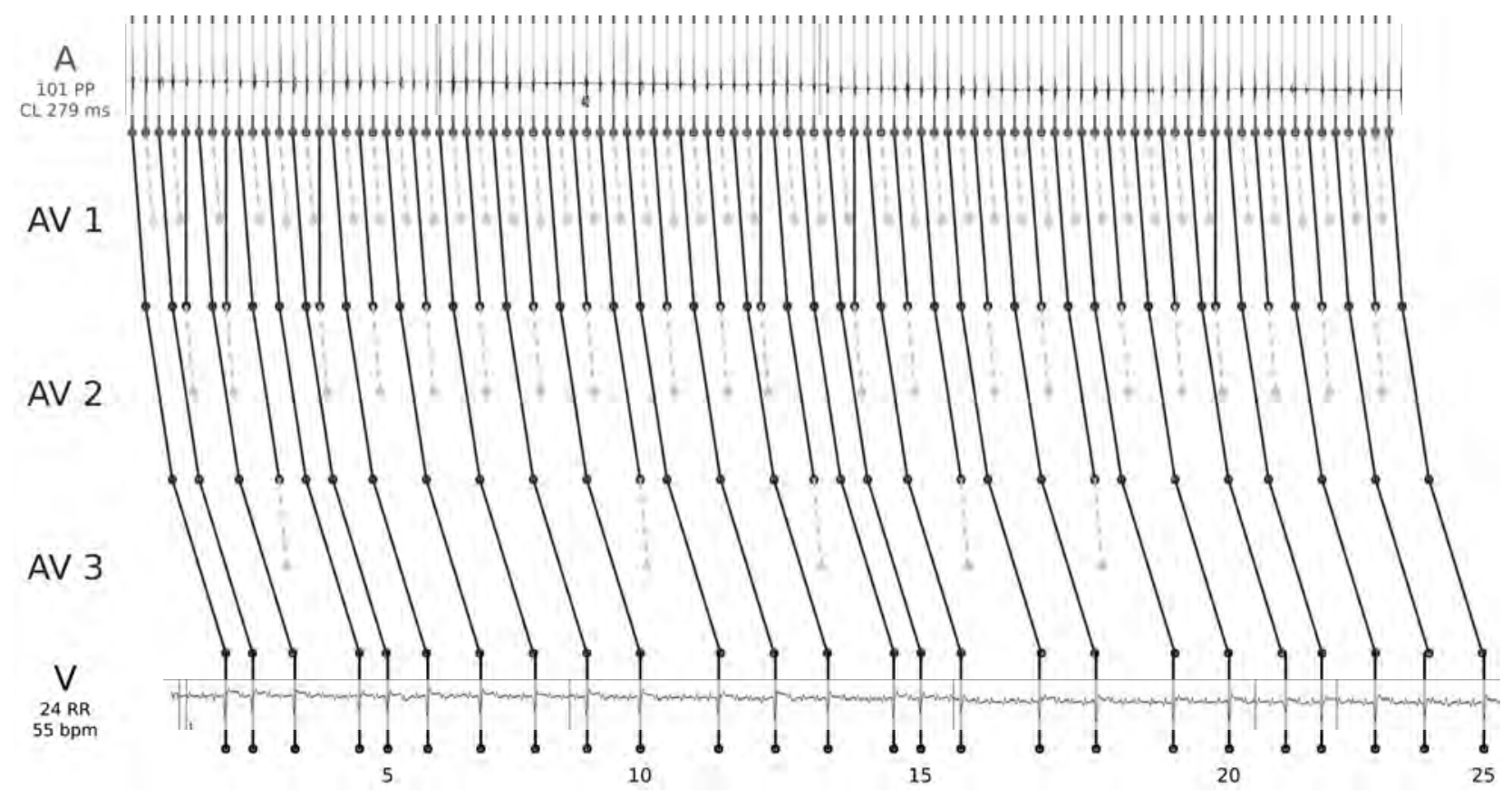

Abbildung 1. Exemplarische Illustration unseres optimierungsgetriebenen Lösungsansatzes aus [15]. Die Inputdaten, die beobachteten ventrikulären Signale (V), werden aus dem Oberflächen-EKG abgelesen (unten im Bild). Als Ergebnis der Optimierung wurden drei Blockierungsebenen und zugehörige Modellparameter berechnet, so dass das errechnete Signal in den Vorhöfen (Atrium, A) regulär ist und die Vorwärtssimulation der Signale nach der untersten Ebene dicht an den Messwerten liegt. Die intrakardialen Messungen werden nur zur Illustration gezeigt (oben), sie sind in der normalen Anwendung gerade nicht verfügbar. 
Kommentar von Jeremi Mizerski, PhD, Herzchirurg und Forscher am Interdisciplinary Center for Mathematical and Computational Modeling, Warschau, Polen (eigene Übersetzung): Das Projekt behandelt das Problem der häufigsten Herzrhythmusstörungen im menschlichen Herzen. Vorhofflimmern und Vorhofflattern sind von Natur aus häufig, haben aber schwerwiegende Konsequenzen. Die medizinische Behandlung der beiden ist komplett unterschiedlich. Die korrekte Diagnose und Differenzierung ist daher von höchster Wichtigkeit, auch aufgrund einer ständig zunehmenden Prävalenz durch unsere alternden Gesellschaften. Die Kraft und Schönheit der modernen Wissenschaft liegen in der Konvergenz und in interdisziplinären Ansätzen. Die enge Verzahnung von medizinischer Forschung und mathematischer Optimierung ist ein exzellentes Beispiel hierfür.

Characteristic [5] betrachtet, wird unser Ansatz mit einem Wert von 0.9 als exzellenter Test klassifiziert. Weitere Details sind in dem paper [21] und der Doktorarbeit [15] zu finden.

Der generelle Ansatz ist patentiert [20] und das spinoff mathe.medical GmbH sucht aktuell nach Möglichkeiten, die mathematische Technologie in die klinische Praxis zu transferieren.

Führung auf der Suche nach dem Ablationspunkt

\section{Klinischer Hintergrund und Optimierungsfragen}

Eine weitere Quelle ungesunder Herzrhythmusstörungen sind vorzeitige Schläge (premature beats, PBs). Sie treten vor allem bei Patienten mit strukturellen Herzkrankheiten auf und oftmals ist eine Katheterablation das Mittel der Wahl. Diese resultiert in Nicht-Konduktivität der ablatierten Bereiche auf der Herzoberfläche und dadurch modifizierten raum-zeitlichen Dynamiken des elektrischen Aktivierungspotentials. Eine offensichtliche Herausforderung ist es, die räumliche Quelle der PBs schnell und verlässlich (um Nebeneffekte zu minimieren) zu identifizieren.

Es wird zunehmend 3 D elektroanatomische Visualisierungssoftware für diesen Zweck eingesetzt. Diese liefert eine graphenbasierte Darstellung der Herzoberfläche mit ${ }_{3}$ D Positionen der Knoten. Das Ablationsgerät kann dabei nicht nur Gewebe vernarben, sondern auch Aktivierungen messen. Dieses wird genutzt, um die lokalen Aktivierungszeiten (LATs) auf Knoten des Graphen abzubilden.

Die Optimierungsaufgabe ist es nun, Knoten für Messungen zu bestimmen, so dass mit einer hohen Wahrscheinlichkeit nur wenige Messungen notwendig sein werden, um den Knoten mit der geringsten LAT zu finden. Bei genauerer Betrachtung erkennt man zwei unterschiedliche Optimierungsaufgaben. Erstens, basierend auf allen bisherigen Messungen den aktuell wahrscheinlichsten Knoten zu bestimmen. Zweitens, solange die damit einhergehende Unsicherheit noch zu hoch ist, den Messknoten auszuwählen, der diese Unsicherheit am meisten reduziert.

\section{Mathematische Modellierung der Dynamik und des Optimierungsproblems}

Wir nutzen zwei Vereinfachungen im Vergleich zu den oben erwähnten first-principle Modellen für elektrische Aktivie- rung. Erstens beschränken wir unsere Betrachtungen auf die Graphenannäherung der Herzoberfläche. Zweitens gehen wir von einer konstanten und homogenen Geschwindigkeit $v$ der elektrischen Aktivierung aus, das heißt, dass die Unterschiede in LATs proportional sind zu den Gewichten des Graphen, den euklidischen Abständen. Das Modell für die LAT $t_{i}$ auf Knoten $i$ ist so gegeben durch

$$
t_{i}=t_{0}+\frac{d_{i k}}{v}
$$

wobei $t_{0}$ die Zeit der frühesten Aktivierung (als offset der relativen Zeitmessungen), $d_{i k}$ die kürzeste Distanz zwischen Knoten $i$ und dem (unbekannten) Quellknoten $k$, und $v$ die Geschwindigkeit sind. Abbildung 2 zeigt einen Vergleich linearer Regressionen für zwei verschiedene Kandidaten für den Quellknoten $k$.
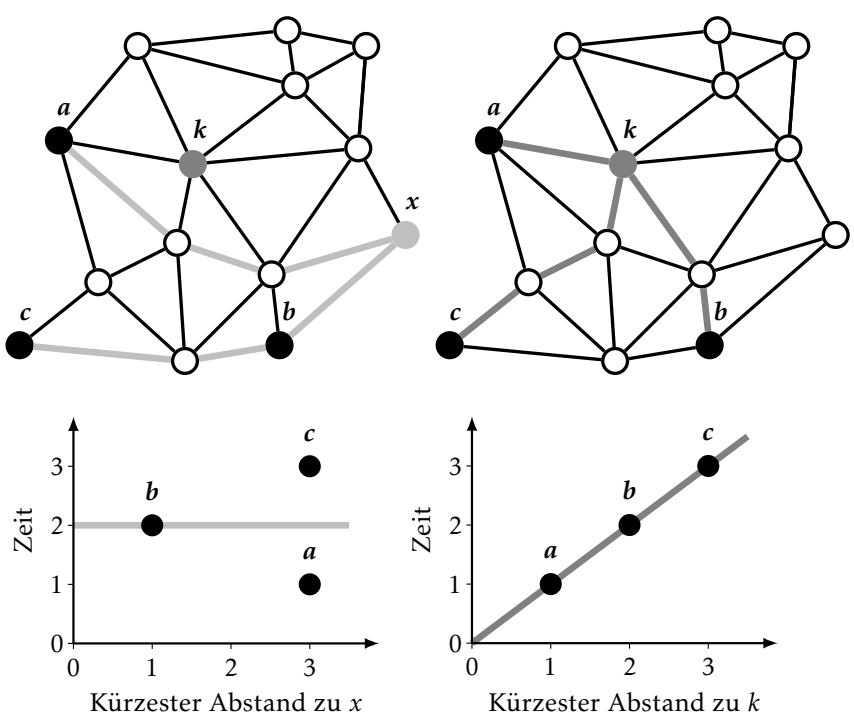

Abbildung 2. (angepasst aus [24]). Lineare Regressionsansätze für einen stark vereinfachten Graphen mit Gewichten 1 für Abstände und Geschwindigkeit. Linke Abbildungen: Als Quelle wird der Knoten $x$ vermutet, der sich von der echten Quelle $k$ (rechte Abbildungen) unterscheidet. Der Vergleich der Messzeiten (die hier den kürzesten Abständen zu der echten Quelle $k$ entsprechen), mit den kürzesten Abständen zwischen $x$ (bzw. $k$ ) und den Messknoten $a, b$, und $c$ führt zu den Abbildungen in der unteren Reihe. Die lineare Regression auf der linken Seite hat ein größeres Residuum, was indiziert dass $x$ kein guter Kandidat für die unbekannte Quelle ist.

\section{Herausforderungen an die Forschung im Bereich der Optimierung}

Man kann die Optimierungsaufgabe auf zwei Arten interpretieren. Erstens als Parameterschätzproblem, bei dem ein „Parameter", der Quellknoten $k \in V$, eine kombinatorische Natur hat. Zweitens als Modelldiskriminierungsproblem, bei dem $\operatorname{card}(V)$ verschiedene Modelle, die zu unterschiedlichen Annahmen an den Quellknoten gehören, darin wetteifern die Messungen zu erklären. Die größte Herausforderung entsteht dabei dadurch, dass die aktuell beste Vermutung für den Quellknoten nicht notwendigerweise der ist, der die meiste Information liefert. Diesen Knoten zu berech- 
Kommentar von Prof. Dr. Eberhard Scholz, Leiter der Elektrophysiologie der Universitätsklinik Heidelberg: Stellen Sie sich vor, Sie wären Mitglied eines Rettungsteams für Lawinenopfer, wohlwissend, dass jede vergehende Minute die Überlebenswahrscheinlichkeit reduziert. Würden Sie hier und dort graben, um das Opfer zufällig zu finden, oder doch lieber einer systematischen Suchroutine mit einem Lawinensignalgeber folgen, um den Vorgang $\mathrm{zu}$ beschleunigen? Interessanterweise zeigt die Lokalisierung von fokalen Quellen von Herzrhythmusstörungen überraschende Parallelen zu der geschilderten Situation auf. Ein kleines Cluster von Herzzellen, das sich an einer unbekannten Stelle des Herzmuskels befindet, sendet ab und zu ein elektrisches Signal. Die meisten Operateure folgen jedoch, anders als ein erfahrenes Rettungsteam, einer eher heuristischen Suche und verlieren dabei wertvolle Zeit. Der in diesem Artikel beschriebene Algorithmus nutzt mathematische Optimierung, um den Operateur auf der Suche nach dem schnellsten Weg zur Quelle zu unterstützen. Das Projekt ist ein tolles Beispiel dafür, wie mathematische Optimierung Medizinern Entscheidungsunterstützung liefern kann und die Tür für eine neue Dimension von Algorithmen in der Elektrophysiologie öffnet.

nen, kann man als Spezialfall der optimalen Versuchsplanung begreifen.

Theoretische Fragestellungen sind die minimale Anzahl von LAT Messungen die notwendig sind, um für ein deterministisches System den Quellknoten durch die metrische Dimension des Graphen zu bestimmen, oder die Verbindung zu der Schranke durch den Satz von Carathéodory. Algorithmische Fragestellungen hängen mit der alternierenden Sequenz von Messungen und Optimierungen und der Frage, wann man von einem „Maximieren des Informationsgehaltes" zu einem „Maximieren der Wahrscheinlichkeit den Quellknoten zu treffen “ übergeht. Lösungen müssen zudem schnell (in Sekundenbruchteilen) berechnet werden, um klinisch in Echtzeit nutzbar zu sein.

Interessanterweise gibt es sehr ähnliche Problemstellungen, bei denen Quellen identifiziert werden müssen. Dazu gehören Verunreinigungen von Trinkwassernetzen oder Pandemien. Der wesentliche Unterschied ist, dass wir sequentiell immer nur eine Messung erhalten und die Möglichkeit haben zu verifizieren, ob ein Knoten der Quellknoten ist (durch die spezifische Form der LAT Kurve in der Quelle).

\section{Lösungsansatz}

Wir induzieren eine Kürzeste-Wege Metrik auf dem Graphen und berechnen alle kürzesten Wege zwischen je zwei Knoten a priori. Bei einer Enumeration aller Kandidatenknoten für die nächste Messung erlaubt diese Metrik den Vergleich linearer Regressionen, wie in Abbildung 2 illustriert. Die aktuell beste Vermutung für den Quellknoten ist der Knoten mit dem kleinsten Residuum. Die VarianzCovarianz Matrix der Parameterschätzung gibt dabei Hinweise auf die Unsicherheit.

In einer ersten Phase des Algorithmus vergleichen wir die berechneten Varianzen und wählen denjenigen Knoten als nächsten Messknoten aus, der den geringsten Wert aufweist. Sollte das Residuum der besten aktuellen Vermutung klein genug sein, wählen wir in einer zweiten Phase diesen Knoten als nächsten Messknoten aus.
(Vorläufige) Ergebnisse und Ausblick

Wir haben eine retrospektive Simulationsstudie mit Daten von 17 Patienten durchgeführt. Die Daten wurden aus dem Carto $3 D$ mapping system exportiert und beinhalteten die Herzgeometrie, alle vom Operateur durchgeführten Messungen und die gemessenen LATs. Unser Algorithmus konnte die durchschnittliche Zahl von LAT Messungen von $42 \pm 7.0$ auf $11 \pm 0.89$ reduzieren, was das enorme Verbesserungspotential für den klinischen Alltag andeutet. Weitere Details sind in dem paper [24] zu finden.

Zukünftige Arbeit sollte sich auf weniger restriktive Annahmen konzentrieren, wie beispielsweise realistischere nicht-konstante Geschwindigkeiten oder vernarbtes Gewebe. Auch eine prospektive Studie, bei der der Algorithmus gemeinsam mit kommerzieller $3 \mathrm{D}$ Software eingesetzt wird, wäre wünschenswert.

\section{Auf dem Weg zur optimalen Behandlung von Blutkrebs}

\section{Klinischer Hintergrund und Optimierungsfragen}

Onkologen und Hämatologen - und oftmals auch betroffene Patienten - müssen zwischen verschiedenen Behandlungsalternativen für Blutkrebs entscheiden. Wenn Chemotherapie eingesetzt wird, beinhaltet diese Entscheidung verschiedene Kombinationen von Medikamenten und Immunboostern, einer zeitlichen Planung der Behandlungen und Dosierungen. Zusätzliche Aspekte, wie das Gleichgewicht zwischen positivem (tötendem) Einfluss auf Krebszellen und negativem (tötendem) Einfluss auf Zellen des Immunsystems, unterstreichen die Bedeutung, den menschlichen Körper als ein komplexes dynamisches System zu begreifen. Offensichtlich wären Simulation (Was würde passieren, wenn?) und Optimierung (Was ist die beste Behandlung?) von enormem Wert, wenn sie verlässlich einsetzbar und für den Spezialfall personalisierbar wären. Bisher haben wir uns in unserer Forschung auf drei verschiedene Arten von Entscheidungsunterstützung für Blutkrebs fokussiert, jeweils motiviert durch praktische medizinische Fragestellungen und verfügbare retrospektive Daten unserer klinischen Partner.

Das erste Projekt befasst sich mit Konsolidierungstherapie für Akute Myeloische Leukämie (AML) bei Erwachsenen. Akut bezieht sich dabei auf plötzliches Einsetzen (im Gegensatz zu chronisch). Myeloisch bezieht sich auf den Ort an dem die Differenzierung und Proliferation der Blutzellen stattfindet, hier im Knochenmark. Und Leukämie bezieht sich auf eine Krankheit die die Leukozyten, die weiBen Blutzellen, betrifft. Die wichtigste chemotherapeutische Behandlung beinhaltet mehrere Zyklen Induktionstherapie, gefolgt von bis zu vier Zyklen Konsolidierungstherapie. Die Therapien unterscheiden sich in den chemotherapeutischen Medikamenten, den Dosierungen, und den Verabreichungszeiten, aber auch in den unterliegenden Zielen. Bei der Induktionstherapie versucht man, alle Blasten (proliferierende Krebszellen) auszulöschen, während die Konsolidierungstherapie beginnt, wenn mit heutigen Messmethoden kaum noch Krebszellen nachgewiesen werden können und man eine Wiederkehr zu verhindern sucht. 
Wir haben das Chemotherapeutikum Cytarabin untersucht, das wohl wichtigste in der Behandlung von AML. Anwendung, zum Beispiel zweimal täglich an den Tagen 1, 3 und 5 eines Konsolidierungszyklus, führt zu einer verzögerten Reduktion der Anzahl der (im Blut) zirkulierenden weißen Blutzellen. Diese Anzahl kann man als Indikator für die Stärke des Immunsystems auffassen, wobei niedrige Werte mit gefährlichen Nebenwirkungen einhergehen. Die Zeitspanne, in der die Anzahl der weißen Blutzellen unterhalb eines kritischen Wertes liegt, bezeichnet man als Leukopenie. Es ist von hoher klinischer Relevanz, die Länge und Tiefe einer Leukopenie vorhersagen zu können. Und sie, wenn möglich, durch eine Modifikation der Behandlung doch noch abwenden zu können. Hier setzt die Optimierung an. Sie kann eingesetzt werden, um mathematische Modelle zu personalisieren und die zukünftige Dynamik der weiBen Blutzellen vorher zu sagen. Sie kann ferner eingesetzt werden, um optimale Versuchspläne zu berechnen, also Messzeiten und Behandlungen, die zu kleinen Konfidenzgebieten von Parameterschätzungen führen. Schlußendlich kann sie eingesetzt werden, um die Verabreichungszeiten und -dosierungen von Chemotherapien zu optimieren.

In einem zweiten Projekt beschäftigen wir uns mit Konsolidierungstherapie für Akute Lymphoblastische Leukämie (ALL) bei Kindern. Bei ALL treten Blasten in der Lymphe (die in den Lymphgefäßen enthaltene Flüssigkeit, die das Zwischenglied zwischen der Gewebsflüssigkeit und dem Blutplasma bildet) und nicht im Knochenmark auf. Die typische Konsolidierungstherapie für Kinder wird fast kontinuierlich gegeben, mit oral verabreichten Medikamenten alle zwei Wochen über Zeiträume von mehreren Jahren. Die klinische Herausforderung besteht darin, die Anzahl der weißen Blutzellen in einem Zielbereich zu halten, indem Verabreichungszeiten und -dosierungen entsprechend angepasst werden. Dieses ist sehr schwierig aufgrund von Unsicherheiten, Verzögerungen und Nichtlinearitäten.

In einem dritten Projekt untersuchen wir Polycythemia vera (PV) bei Erwachsenen. Bei PV produziert das Knochenmark zu viele Erythrozyten (rote Blutzellen). Der Anstieg in der Anzahl der roten Blutzellen führt zu einer Verdickung des Blutes, welche durch die Gefahr von Thrombosen tödlich sein kann. Da die Krankheit (noch) nicht geheilt werden kann, fokussiert sich die Behandlung primär auf die Symptome. Sie besteht im wesentlichen, wie im Mittelalter, aus einem Aderlass. Ein solcher macht hier tatsächlich großen Sinn, da sich die roten Blutzellen innerhalb von Wochen regenerieren, während das Blutplasma dieses innerhalb von Stunden tut. Daher nimmt der Hämatokrit (Prozentsatz von roten Blutzellen im Blut) durch einen Aderlass ab. Nebeneffekte wie Schwindel, Müdigkeit und Kopfschmerzen kennt jeder Blutspender. Eine spannende klinische Frage ist, ob man personalisierte Simulationen und Optimierung nutzen kann um Aderlässe optimal zu planen. Optimal bezieht sich hierbei auf die Anzahl in einem bestimmten Zeitraum, die Zeiträume in denen der Hämatokrit in einem kritischen Bereich liegt, aber auch in Kollisionen mit wichtigen persönlichen und beruflichen Terminen, die häufig von Patienten als wesentliche Einschränkung ihrer Lebensqualität wahrgenommen werden.
Mathematische Modellierung der Dynamik und des Optimierungsproblems

Krebsdynamiken können auf vielen verschiedenen Ebenen modelliert werden [3], selbst im Falle von Blutkrebs, bei dem räumliche Aspekte des Tumorwachstums nicht berücksichtigt werden müssen. Für die oben skizzierten Optimierungsansätze ist die mathematische Modellierung der Hämatopoese (Blutbildung durch Differentiation und Reifung ausgehend von blutzellbildenden Stammzellen) besonders wichtig. In der Literatur gibt es einen Fundus von durchdachten Modellen mit komplexen Pfadwegen, Verzögerungen, spieltheoretischen Ansätzen zum Wettbewerb zwischen Zellen, oder partiellen Differentialgleichungen mit dem Reifegrad der Zellen als räumlicher Dimension. Solche komplexen Modelle haben normalerweise viele Modellparameter, die aufgrund der vorliegenden Messdaten nicht identifiziert werden können und eignen sich nur bedingt für optimierungsgetriebene Entscheidungsunterstützung in Echtzeit.

Wir entwickeln, analysieren und nutzen Modelle, die nur die wichtigsten Aspekte der Blutzelldynamik beinhalten und verschiedene physiologische Effekte in vereinfachenden Ausdrücken zusammenfassen. Zudem vernachlässigen wir oftmals die Dynamik der Krebszellen, da diese in den beschriebenen Umständen eh nicht messbar sind. Wenn Medikamente verabreicht werden, sind die Pharmakokinetik (PK; was der Organismus mit dem Medikament macht) und die Pharmakodynamik (PD; was das Medikament mit dem Organismus macht) wichtige weitere Modellierungsbausteine. Wir nutzen unter anderem Kompartimentenmodelle. Hier werden Zellen unterschiedlichen Reifegrades, die gemeinsames Verhalten wie Proliferation oder Differenzierung zeigen, in Kompartimenten zusammengefasst. Im AML Projekt nutzen wir Mengen $x_{1}$ und $x_{2}$ des Chemotherapeutikums Cytarabin in zwei PK Kompartimenten und Anzahlen $x_{\mathrm{pr}}$ von proliferierenden, $x_{\mathrm{tr}, 1}, \ldots, x_{\mathrm{tr}, n_{\mathrm{tr}}}$ von differenzierenden Zellen in $n_{\mathrm{tr}}$ Kompartimenten, und $x_{\text {ma }}$ von zirkulierenden weißen Blutzellen. Die zeitabhängige Cytarabinverabreichung wird mit $u(t)$ bezeichnet. Das System von gewöhnlichen Differentialgleichungen für Hämatopoese, Pharmakokinetik und Pharmakodynamik basiert auf dem Goldstandard-Modell für Myelosuppression [9] und schreibt sich als

$$
\begin{aligned}
\dot{x}_{1}(t) & =-\left(k_{10}+k_{12}\right) x_{1}(t)+k_{21} x_{2}(t)+u(t) \\
\dot{x}_{2}(t) & =k_{12} x_{1}(t)-k_{21} x_{2}(t) \\
\dot{x}_{\mathrm{pr}}(t) & =-k_{\mathrm{tr}} x_{\mathrm{pr}}(t)+F\left(x, k_{\mathrm{tr}}, \gamma, B, \text { slope }\right) x_{\mathrm{pr}}(t) \\
\dot{x}_{\mathrm{tr}, 1}(t) & =k_{\mathrm{tr}} x_{\mathrm{pr}}(t)-k_{\mathrm{tr}} x_{\mathrm{tr}, 1}(t) \\
\dot{x}_{\mathrm{tr}, 2}(t) & =k_{\mathrm{tr}}\left(x_{\mathrm{tr}, 1}(t)-x_{\mathrm{tr}, 2}(t)\right) \\
& \cdots \\
\dot{x}_{\mathrm{tr}, n_{\mathrm{tr}}}(t) & =k_{\mathrm{tr}}\left(x_{\mathrm{tr}, n_{\mathrm{tr}}-1}(t)-x_{\mathrm{tr}, n_{\mathrm{tr}}}(t)\right) \\
\dot{x}_{\mathrm{ma}}(t) & =k_{\mathrm{tr}} x_{\mathrm{tr}, n_{\mathrm{tr}}}(t)-k_{\mathrm{ma}} x_{\mathrm{ma}}(t)
\end{aligned}
$$

mit einer Funktion $F$, die den pharmakodynamischen Effekt von Cytarabin und mögliche Feedbackmechanismen auf die proliferierenden Zellen modelliert. Die Modellparameter 


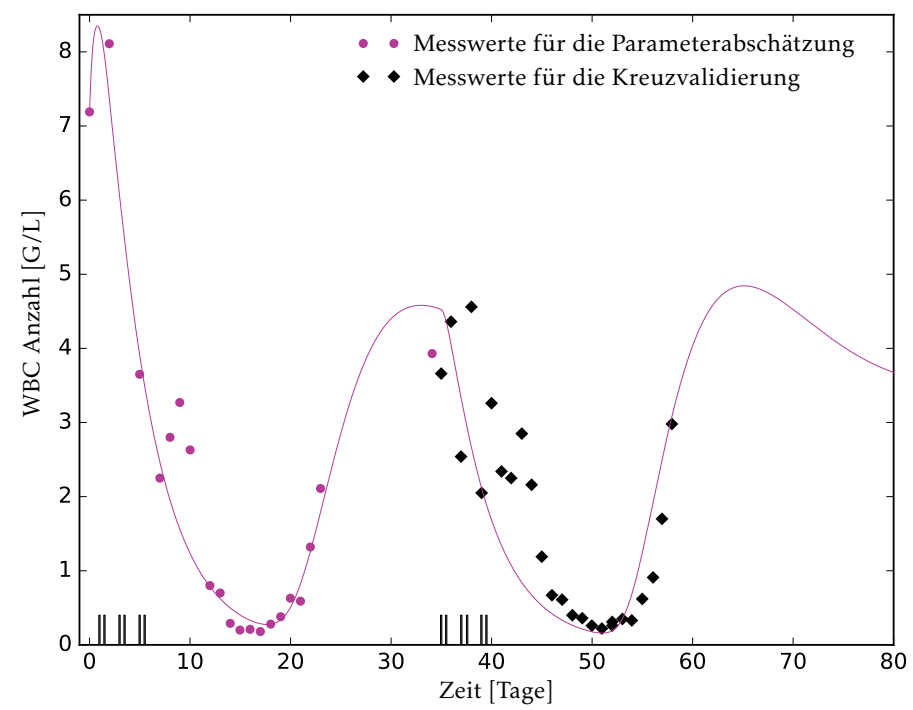

Abbildung 3. Beispielhafter Datensatz für einen AML Patienten aus [12]. Die Verabreichungen der Chemotherapie sind auf der $\mathrm{x}$-Achse angedeutet. Die Punkte sind Anzahlen weißer Blutzellen (WBC). Die Trajektorie zeigt die Lösung $x_{\mathrm{ma}}(\cdot)$ eines personalisierten Modelles vom Typ (4), basierend auf den Messungen des ersten Konsolidierungszyklus. Eine Kreuzvalidierung mit den Messdaten eines zweiten Konsolidierungszyklus gibt einen Hinweis auf die hohe prädiktive Genauigkeit des Modelles.

und Anfangswerte, die genutzt werden können um (4) zu personalisieren, sind

$$
p=\left(B, k_{\mathrm{tr}}, \gamma \text {, slope, } x_{\mathrm{pr}}\left(t_{0}\right), x_{\mathrm{tr}}\left(t_{0}\right), x_{\mathrm{ma}}\left(t_{0}\right)\right),
$$

während wir alle anderen aus Gründen der Identifizierbarkeit auf Literaturwerte aus in vitro Studien fixieren. Abbildung 3 zeigt ein Beispielergebnis mit klinischen Daten. Das Modell wurde an Messungen von weißen Blutzellen $\left(\approx x_{\mathrm{ma}}\right)$ aus einem Konsolidierungszyklus angepasst. Die Daten des folgenden Zyklus wurden mit der Vorhersage als Kreuzvalidierung verglichen.

Die Modelle für ALL und PV [23] sind strukturell ähnlich zu (4) Die Vorhersagegenauigkeit von (4) ist recht hoch, und erstaunlich stabil gegenüber Änderungen in der Modellierung von $F$. Während dies erfreulich ist bezüglich der Simulation eines fixierten Behandlungsplans, ist das Modell nicht notwendigerweise geeignet, um verschiedene Dosierungen und Verabreichungszeiten zu evaluieren oder gar zu optimieren. Die zusammenführende Natur des mathematischen Modelles führt nämlich zu Modellparametern, die nicht nur für den Patienten personalisiert sind, sondern auch für den verabreichten Behandlungsplan. Wir entwickeln aktuell Modelle, die in der Lage sind für verschiedene Behandlungspläne genaue Vorhersagen zu machen. Eine Verifikation mit Daten ist offensichtlich besonders herausfordernd, da identische Wiederholungen unterschiedlicher Behandlungspläne in der Realität nicht möglich sind.

Wenn man die Verfügbarkeit eines validierten personalisierten Modelles voraussetzt, ergeben sich eine Vielzahl von Optimierungsfragen. Sind hohe oder niedrige Dosierungen (oder sogenannte singuläre Bögen, also sich zeitlich verändernde Dosierungen) vorzuziehen? Welchen Einfluss haben die Verabreichungszeiten der Chemotherapie? Wie wichtig ist der Abstand zwischen zwei Konsolidierungszyklen? Was sind gute Zeitpunkte, um Immunbooster zu geben? Wie beeinflussen all diese Änderungen die Krebszellen? Wie sehr hängen die Ergebnisse von der Wahl der Zielfunktion $\mathrm{ab}$ ? Wenn beispielsweise $x_{\text {ma }}$ so groß wie möglich sein soll, würde man eher $x_{\text {ma }}\left(t_{\mathrm{f}}\right)$ oder $\int_{0}^{t_{\mathrm{f}}} x_{\mathrm{ma}}(\tau) \mathrm{d} \tau$ maximieren?

Interessanterweise kann Optimierung auch genutzt werden, um das Potential von modifizierten Behandlungen zu evaluieren und sicher zu stellen, dass der Aufwand sich überhaupt lohnt. In [8] schlugen wir vor, die Zielfunktion zu minimieren und zu maximieren. Wenn die Gesamtmenge des Chemotherapeutikums durch Nebenbedingungen fixiert wird, beantwortet der Vergleich der beiden Zielfunktionswerte die Frage, wie sehr sich die beste von der schlechtesten Behandlung unterscheidet. Wenn dieser Unterschied nicht wesentlich größer ist als die intrisische Unsicherheit, macht der Optimierungsansatz wenig Sinn.

Die Verfügbarkeit mathematischer Modelle erlaubt ein weiteres interessantes Gedankenexperiment. Was wäre, wenn einige Modellparameter Steuerfunktionen wären? In unserer abstrakten Problemformulierung lassen sich konstante Parameter leicht durch zeit-abhängige optimierbare Funktionen ersetzen. Optimale Lösungen dieser künstlichen Probleme könnten Hinweise für Pharmakologen und Onkologen liefern, welche Arten von Medikamenten notwendig sind, um die nichtlineare Dynamik auszunutzen.

\section{Herausforderungen an die Forschung im Bereich der Optimierung}

Die größte Herausforderung liegt in der angenommenen Verfügbarkeit von validierten personalisierten Modellen, die offensichtlich falsch ist. Personalisierte Modelle mit kleinen Standardabweichungen der Parameter (und damit kleinen Unsicherheiten von Vorhersagen) benötigen eine bestimmte Anzahl von Messungen, die erst während einer Behandlung durchgeführt werden. Damit treten neue Fragen auf. Was ist eine gute Behandlungsstrategie, wenn die Unsicherheit des Modelles noch hoch ist? Wann sollten Messungen gemacht werden, um genaue Parameterschätzungen zu erhalten? Wie beeinflussen der Chemotherapie- bzw. Aderlassplan die Konfidenzgebiete von nachfolgenden Zustands- und Parameterschätzungen? Macht es vielleicht Sinn, früh geringe Dosen von Chemotherapie zu geben auch wenn kein direkter medizinischer Nutzen zu erwarten ist, sehr wohl aber ein indirekter durch sicherere Modelle und verlässlichere Vorhersagen? Der Ansatz der optimalen Versuchsplanung, der ein Kriterium der Varianz-Covarianz Matrix minimiert, führt zu genauen Zustands- und Parameterschätzungen, kann aber ein schlechtes Behandlungsergebnis verursachen. Zielsetzung der sogenannten dual control ist dagegen eine Optimierung des Endergebnisses der Behandlung, deren Ausgestaltung auf Modellvorhersagen beruht, die wiederum von genau dieser Behandlung abhängen.

Unsere Algorithmen schlagen Behandlungspläne vor, die sowohl zu einer genauen Patienten-spezifischen Personalisierung von Modellen in Echtzeit als auch zu den gewünschten klinischen Ergebnissen führen. Obwohl mehrere Ansätze in der Literatur vorgeschlagen wurden, siehe 
beispielsweise [13], gibt es viele offene theoretische und algorithmische Fragen in der dual control.

Ebenfalls wichtig sind die Aspekte von dünnbesetzten Steuerungen (da Patienten so wenig Zeit wie möglich im Krankenhaus verbringen wollen und sollten), logischen Implikationen die aus rechtlichen oder klinischen Erwägungen resultieren, und globale Optimalität.

\section{Lösungsansatz}

Unsere Algorithmen basieren auf dem first-discretize, thenoptimize Ansatz aus der Schule von Georg Bock. Wir haben diesen in verschiedene Richtungen erweitert. Auf einer oberen Ebene entwickeln wir neue Konzepte und Algorithmen für dual control [13] um mit Optimierung iterativ neue personalisierte Modelle, optimale Messzeiten und optimale Behandlungspläne zu berechnen. Die Bausteine sind Methoden aus der Zustands- und Parameterschätzung auf bewegten Zeithorizonten, robust optimale Versuchsplanung, Modelldiskriminierung, nichtlineare modellprädiktive Regelung, gemischt-ganzzahlige optimale Steuerung und dual control. Wir haben unter anderem einen neuen Algorithmus und Löser zur sequentiellen quadratischen Optimierung entwickelt, der Blockstrukturen ausnutzt, wie sie bei Optimalsteuerungsproblemen auftreten, [11]. Die algorithmischen Entwicklungen werden normalerweise durch konkrete klinische Daten und Fragestellungen wie in $[12,18]$ stimuliert. Um Informationen von anderen Patienten nutzen zu können, erweitern wir unsere Methoden auch auf so genannte gemischte Modelle, die Modellparameter für eine ganze Population und die inter-individuellen Varianzen liefern.

\section{(Vorläufige) Ergebnisse und Ausblick}

Bei AML wird durch die optimale Wahl von 20-30 Messzeitpunkten (fast täglich) für einen Konsolidierungszyklus durch Versuchsplanung die Unsicherheit der Parameterschätzungen um ungefähr $50 \%$ reduziert [12]. Optimale Messungen eines Konsolidierungszyklusses führen zu Standardabweichungen der Parameter von Pi mal Daumen 10\% und damit hinreichend genauen Vorhersagen der folgenden Zyklen.

Diese Vorhersagen können für eine Optimierung genutzt werden. Genau genommen, sollten sie für die Optimierung genutzt werden. Selbst für sehr einfache Modelle ohne Pharmakokinetik ergeben sich nicht-intuitive optimale Steuerungen, die den entscheidenden Unterschied zu Standardtherapien ausmachen können, [8]. Dieses Zwischenergebnis scheint sich nach aktuellem Stand auch für die komplexeren Prozesse bei AML, ALL und PV zu bestätigen (aktuell zur Veröffentlichung eingereichte Arbeiten). Wir haben hier jeweils neue mathematische Modelle entwickelt, die mit klinischen Daten personalisiert und kreuzvalidiert wurden. Mit diesen Modellen konnten wir erklären, wann und warum kompakte Chemotherapie Behandlungspläne besser sind und eine neue dynamische Stratifizierung von Blutkrebspatienten vorschlagen. Unsere Analysen zeigen viele Fälle, bei denen ein optimierter Behandlungsplan zumindest laut Simulation deutlich bessere Ergebnisse (z. B. Vermeidung einer Leukopenie) erzielt hätte.
Kommentar von Prof. Dr. Thomas Fischer, Direktor der Klinik für Hämatologie und Onkologie, Universitätsklinik Magdeburg: Simulation und Optimierung vermögen die Behandlung von Blutkrebs zu verändern. Beispielsweise könnten verlässliche Voraussagen genutzt werden um AML Patienten in der Konsolidierungstherapie besser zu versorgen. Analysen der Chemotherapie-induzierten tiefen Leukopenien und Anwendung optimierter Behandlungspläne könnten schwere infektiöse Komplikationen und damit Verzögerungen nachfolgender Zyklen vermeiden. Die Dichte von Chemotherapiezyklen würde erhöht, und damit würden tiefere Remissionen und geringere Wahrscheinlichkeiten eines Rezidivs ermöglicht werden. Dies könnte schlussendlich zu verbesserten Überlebensraten führen.

Aus meiner persönlichen Sicht sind die vorgeschlagenen Modelle, Algorithmen und Resultate kleine, aber wichtige Schritte auf dem Weg zu einem personalisierten Echtzeitalgorithmus, der die nichtlineare Dynamik und kombinatorische Komplexität von Blutkrebsbehandlungen nutzt. Viele Fragen und Anwendung in prospektiven klinischen Studien sind allerdings noch offen.

\section{Ausblick: Optimierung für das Training Klinischer Entscheidungen}

Lehre und Ausbildung werden sich sicherlich zukünftig stark ändern, beispielsweise durch virtuelle Realität oder künstliche Intelligenz. Setzt man die Verfügbarkeit von mathematischen Modellen und maßgeschneiderten Optimierungsalgorithmen voraus, ist es eine natürliche Frage, ob man auch mit ihnen die Ausbildung verbessern kann. Mit Simulatoren wird schon jetzt erfolgreich gelernt wie man fährt, fliegt, Energie-effiziente Häuser, Autos oder Flugzeuge baut, große Gas- und Wassernetzwerke steuert oder Ticketpreise festsetzt. Nach meiner Auffassung wird dieses Konzept auch in der Medizin tragen. Ungewisser ist hingegen, ob auch Optimierung einen Mehrwert leisten kann.

Das Treffen klinischer Entscheidungen kann als ein Spezialfall des Komplexen Problemlösens (complex problem solving, CPS) betrachtet werden, einer in der Psychologie beheimateten Forschungsrichtung. Probleme werden hier (ähnlich wie in der Optimierung) als komplex angesehen, wenn sie eine oder mehrere der Eigenschaften hohe Dimension, hohe nichtlineare Kopplung, beträchtliche Eigendynamik, Unsicherheiten und Mehrzieligkeit beinhalten. Die wesentliche Intention der Forschungsrichtung ist es, zu verstehen, wie bestimmte Variablen den Lösungsprozess beeinflussen. Hierbei werden persönliche Variablen wie Intelligenz, Arbeitsgedächtnis, Wissen, und Emotionsregulation von situationsgebundenen Variablen wie Zielspezifikation, Feedback oder zeitlichen Limitierungen unterschieden. Einige allgemeine Fragen sind dabei, warum es für Menschen so schwer ist, Folgen von Entscheidungen einzuschätzen und abzuwägen, oder welchen Einfluss beispielsweise kognitive Repräsentation oder Emotionen haben.

Ein wesentliches methodologisches Hindernis, um diese Fragen zu beantworten, ist die Frage wie Performanz in komplexen Problemen überhaupt gemessen werden kann. 
In [19] haben wir einen neuen verlässlichen und objektiven Performanzindikator vorgeschlagen, am Beispiel des tailorshop. Bei diesem ist die Aufgabe, innerhalb von $n$ Runden den Wert eines Hemden produzierenden Unternehmens durch Entscheidungen in den Bereichen Produktion, Materialmanagement, Marketing und Personal zu maximieren. Die "Fruchtfliege" der CPS Forschung ist in Abbildung 4 visualisiert. Wir haben erstmals eine derart komplexe $\mathrm{Mi}$ krowelt als kompaktes mathematisches Modell formuliert. Basierend darauf optimieren wir für jede gespielte Runde und starten mit genau den gleichen Bedingungen wie die Teilnehmer/innen der Studie sie in der Runde $n_{s}$ (durch ihre früheren Entscheidungen) hatten. Die Unterschiede der optimalen Zielfunktionswerte der Runden $n_{s}$ und $n_{s}+1$ bezeichnen wir als How-much-is-still-possible-Funktion. Sie gibt an, wie gut die Performanz im Vergleich zu optimalen Entscheidungen war. Dieses Performanzmaß ist objektiv und gibt zudem Einblick in die zeitliche Evolution der Performanz, d.h. man kann genau nachvollziehen in welchen Runden kritische Fehler begangen wurden.

Dieser neue methodische Ansatz wurde für experimentelle Studien genutzt $[1,2,19]$. In [2] wurde gezeigt, dass Teilnehmer/innen die ein negatives Feedback erhalten, bessere Performanz zeigen als diejenigen mit positivem Feedback. In $[1,19]$ wurde zusätzlich die Fähigkeit zur Emotionsregulation betrachtet. Es konnte ein Zusammenhang zwischen dieser und dem Feedback gezeigt werden: Teilnehmer/innen mit hoher Fähigkeit zur Emotionsregulation schnitten bei negativem Feedback besser ab, diejenigen mit einer niedrigen Fähigkeit dagegen bei positivem Feedback.

In $[6,7]$ befassten wir uns mit der Frage, ob optimale Lösungen auch zur Verbesserung der Ausbildung genutzt werden können. Wir untersuchten in einer online Studie zwei Haupthypothesen: Optimierungsgestütztes Feedback in einer Trainingsphase verbessert die Performanz in darauf folgenden Performanzphasen und es erhöht das Modellwissen im Vergleich $\mathrm{zu}$ einer Kontrollgruppe. Beide Hypothesen wurden durch Daten von mehr als 1 oo Teilnehmern bestätigt. Wir nutzten insbesondere graphische

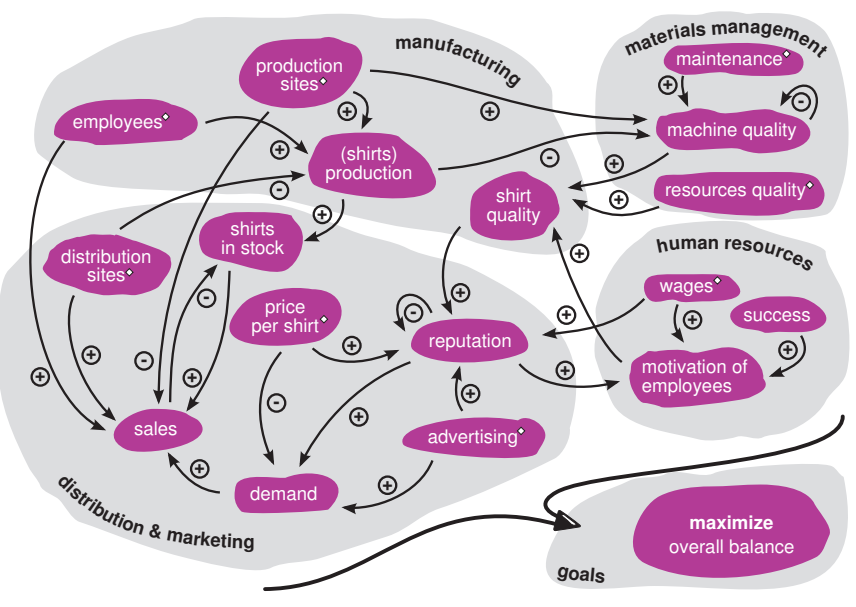

Abbildung 4. Schema der Steuerungen (angedeutet mit einem Diamanten) und Zustandsvariablen in jeder Runde der tailorshop Mikrowelt [6]. Pfeile zeigen Abhängigkeiten. Einige Entscheidungen sind ganzzahlig, einige Zusammenhänge sind nichtlinear.
Kommentar von Prof. Dr. Joachim Funke, Psychologisches Institut, Universität Heidelberg: Europas Entscheidungsträger/innen in Politik, Industrie, Technologie und Wissenschaft sind mit der Herausforderung zunehmender Komplexität konfrontiert. Daher hat das OECD PISA Konsortium Complex Problem Solving (CPS) Kompetenzen als wesentlichen Teil der menschlichen Ausbildung definiert. Für die Analyse und das Training von CPS Kompetenzen braucht es einen synergetischen Ansatz. Zum einen müssen die unterliegenden kognitiven Prozesse besser verstanden werden. Zum anderen werden mächtige Optimierungswerkzeuge benötigt a) um Trainingsumgebungen zu entwickeln, die bestimmte Eigenschaften haben, b) um die Performanz von Teilnehmer/innen zu analysieren und c) um optimierungsgetriebenes feedback geben zu können.

Der Einfluß der Optimierung auf die psychologische Forschung ist immens. Zum ersten mal seit dem Aufkommen der CPS Forschung in den späten 1970er Jahren erlaubt die Optimierung komplexer Probleme die Evaluation der Performanz in absoluten Einheiten. Dieses ist ein fundamentaler Forschritt gegenüber früheren Ansätzen, die nur relative Aussagen bezogen auf die teilnehmende Studienpopulation machen konnten. Die Evaluation individueller Entscheidungen im zeitlichen Verlauf und neue Trainingsmethoden eröffnen zudem gänzlich neue und spannende Möglichkeiten für die zukünftige CPS Forschung.

Visualisierungen optimaler Entscheidungen und von Lagrangemultiplikatoren getroffener Entscheidungen. Die Ergebnisse deuten das große Potential an, das Optimierung auch für das Lernen hat, allerdings sind viele Fragen wie beispielsweise die Frage nach guten Visualisierungen, das Problem lokal optimaler Lösungen oder das Messen der Exploration von Mikrowelten noch offen. Für die zu lösenden gemischt-ganzzahligen nichtlinearen Optimierungsprobleme haben wir spezifische Dekompositions- und Unterschätzungstechniken entwickelt, die Lösungen innerhalb weniger Sekunden produzieren.

\section{Zusammenfassung}

Wir haben in mehreren Fallstudien die spannenden Wechselwirkungen zwischen Optimierung und klinischer Entscheidungsfindung beleuchtet.

Personalisierte Medizin wird häufig gleichgesetzt mit einer Stratifizierung durch Genomics. Ich glaube an das große Potential einer komplementären dynamischen Stratifikation, die auf individuell gefitteten mathematischen Modellen und durch Optimierung gewonnenen Einsichten basiert.

Der größte Einfluß von mathematischen Techniken im klinischen Alltag dürfte in einem Paradigmenwechsel liegen. Mediziner werden zukünftig die Auswirkungen ihrer Entscheidungen auf einem Computer simulieren und optimierte Lösungsvorschläge als Alternativen in Betracht ziehen können, mit offensichtlichem Mehrwert.

\section{Danksagung}

Finanzielle Unterstützung der Projekte erfolgte durch den ERC Consolidator Grant MODEST (647573) und durch die Deutsche Forschungsgemeinschaft (314838170, GRK 2297 MathCoRe). 


\section{Literatur}

[1] C. Barth. The Impact of Emotions on Complex Problem Solving Performance and Ways of Measuring this Performance. PhD thesis, Ruprecht-Karls-Universität Heidelberg, 2010.

[2] C. Barth and J. Funke. Negative affective environments improve complex solving performance. Cognition and Emotion, 24:12591268,2010

[3] J. Clairambault. Modelling physiological and pharmacological control on cell proliferation to optimise cancer treatments. Mathematical Modelling of Natural Phenomena, 4(3):12-67, 2009.

[4] P. Deuflhard, M. Grötschel, D. Hömberg, U. Horst, J. Kramer, V. Mehrmann, K. Polthier, F. Schmidt, C. Schütte, M. Skutella, and J. Sprekels, editors. MATHEON - Mathematics for Key Technologies, volume 1. 2014.

[5] I. G. Duncan. Healthcare Risk Adjustment and Predictive Modeling. ACTEX Publications, 2011.

[6] M. Engelhart. Optimization-based Analysis and Training of Human Decision Making. PhD thesis, University Heidelberg, 2015.

[7] M. Engelhart, J. Funke, and S. Sager. A web-based feedback study on optimization-based training and analysis of human decision making. Journal of Dynamic Decision Making, 3(1), 2017.

[8] M. Engelhart, D. Lebiedz, and S. Sager. Optimal Control for Cancer Chemotherapy ODE Models: Potential of optimal schedules and choice of objective function. Mathematical Biosciences, 229(1):123-134, 2011.

[9] L. Friberg, A. Henningsson, H. Maas, L. Nguyen, and M. Karlsson. Model of Chemotherapy-Induced Myelosuppression with Parameter Consistency Across Drugs. Journal of Clinical Oncology, 20:4713-4721, 2002.

[10] M. Grötschel, K. Lucas, and V. Mehrmann, editors. Production Factor Mathematics. 2010.

[11] D. Janka, C. Kirches, S. Sager, and A. Wächter. An SR1/BFGS SQP algorithm for nonconvex nonlinear programs with blockdiagonal Hessian matrix. Mathematical Progamming Computation, 8(4):435-459, 2016.

[12] F. Jost, K. Rinke, T. Fischer, E. Schalk, and S. Sager. Optimum experimental design for patient specific mathematical leukopenia models. In Proceedings of the Foundations of Systems Biology in Engineering (FOSBE) Conference, volume 49, pages 344-349. Magdeburg, Germany, Elsevier, 2016.
[13] F. Jost, S. Sager, and T. Le. A feedback optimal control algorithm with optimal measurement time points. Processes, 5(10):1-19, 2017.

[14] W. Jäger and H.-J. Krebs, editors. Mathematics: Key technology for the future; joint projects between universities and industry. Springer, Berlin, 2003.

[15] F. Kehrle. Inverse Simulation for Cardiac Arrhythmia. PhD thesis, Otto-von-Guericke University Magdeburg, 2018.

[16] D. Noble. A modification of the Hodgkin--Huxley equations applicable to purkinje fibre action and pacemaker potentials. The Journal of Physiology, 16o(2):317-352, 1962.

[17] D. Noble, A. Garny, and P. J. Noble. How the Hodgkin-Huxley equations inspired the cardiac physiome project. The Journal of Physiology, 59o(Pt 11):2613-2628, 2012.

[18] K. Rinke, F. Jost, R. Findeisen, T. Fischer, R. Bartsch, E. Schalk, and S. Sager. Parameter estimation for leukocyte dynamics after chemotherapy. In Proceedings of the Foundations of Systems Biology in Engineering (FOSBE) Conference, volume 49, pages 44-49. Magdeburg, Germany, Elsevier, 2016.

[19] S. Sager, C. Barth, H. Diedam, M. Engelhart, and J. Funke. Optimization as an Analysis Tool for Human Complex Problem Solving. SIAM Journal on Optimization, 21(3):936-959, 2011.

[20] E. Scholz, S. Sager, and H. Katus. A system and computer program product for automatically distinguishing atrial flutter from atrial fibrillation, 2016.

[21] E. P. Scholz, F. Kehrle, S. Vossel, A. Hess, E. Zitron, H. A. Katus, and S. Sager. Discriminating atrial flutter from atrial fibrillation using a multilevel model of atrioventricular conduction. Heart Rhythm Journal, 11(5):877-884, 2014.

[22] A. Shiyovich, A. Wolak, L. Yacobovich, A. Grosbard, and A. Katz. Accuracy of diagnosing atrial flutter and atrial fibrillation from a surface electrocardiogram by hospital physicians: Analysis of data from internal medicine departments. The American Journal of the Medical Sciences, 340(4):271-275, 2010.

[23] M. Tetschke, P. Lilienthal, T. Pottgiesser, T. Fischer, E. Schalk, and S. Sager. Mathematical modeling of RBC count dynamics after blood loss. Processes, 6(9):157-185, 2018.

[24] T. Weber, H. Katus, S. Sager, and E. Scholz. Novel algorithm for accelerated electroanatomical mapping and prediction of earliest activation of focal cardiac arrhythmias using mathematical optimization. Heart Rhythm, 14(6):875-882, 2017.

Prof. Dr. Sebastian Sager, Fakultät für Mathematik, Otto-von-Guericke Universität Magdeburg, Institut für Mathematische Optimierung (IMO), Universitätsplatz 2, 39106 Magdeburg

sager@ovgu.de

Sebastian Sager ist seit 2012 Professor für Algorithmische Optimierung an der Otto-von-Guericke Universität Magdeburg. Er studierte Mathematik in Heidelberg, wo er auch promoviert wurde und sich habilitierte. Seine Forschung in der Schnittstelle zwischen Medizin und Mathematik wurde 2015 mit einem Consolidator Grant des European Research Council ausgezeichnet. Er ist zudem Sprecher des Graduiertenkollegs 2297 „Mathematische Komplexitätsreduktion" der Deutschen Forschungsgemeinschaft.

Eine frühere Version dieses Beitrags ist in Optima 104 (2018), S. 1-8, unter dem Titel „Optimization and clinical decision support“ erschienen. 